\title{
Competitive exclusion in Cladocera through elevated mortality of adults
}

\author{
Vladimir Matveev' and Wilfried Gabriel \\ Max-Planck-Institut für Limnologie, Postfach 165, D-24302 Plön, FRG \\ 'Present address and address for correspondence: The Murray-Darling \\ Freshwater Research Centre, PO Box 921, Albury 2640, Australia
}

\begin{abstract}
The population dynamics of two cladocerans, Ceriodaphnia pulchella and Diaphanosoma brachyurum competing under laboratory conditions in lake water was analysed using crosscorrelations. Both mixed and isolated populations of the two cladocerans showed delayed densitydependence in the death rates of juveniles and adults as well as in fecundity rate. The regressions for each of the three rates on total density of competitors were compared between the two species. There were no significant differences in the slopes of regressions for fecundity rates and the death rates of juveniles. However, in the inferior competitor (Diaphanosoma) which went extinct in all treatments, the death rate of adults increased with total density much more quickly than in the superior competitor (Ceriodaphnia). The intraspecific comparisons indicated that while Ceriodaphnia adults survived better than juveniles under conditions of crowding, in Diaphanosoma, juveniles were better survivors than adults. These data suggest that the contention of higher vulnerability of cladoceran juveniles than adults to starvation and crowding may prove to be not a universal phenomenon.
\end{abstract}

\section{Introduction}

In Cladocera, juveniles are usually considered more sensitive to crowding and starvation than adults, and this contention has been used to predict the outcome of competition. For example, Neill (1975) has found that adults of Ceriodaphnia outcompeted Daphnia in laboratory cultures through suppression of its juveniles. Lynch (1978) gave evidence of higher sensitivity to starvation of juvenile Daphnia during co-exploitative interactions with other cladocerans in the lake. Tessier et al. (1983) have found in laboratory experiments that starvation time in Daphnia is generally shorter in juveniles than adults; however, it varied strongly with the amount of lipid reserves accumulated in the body of animals. The contention of higher sensitivity of juveniles to starvation was finally incorporated in a model, predicting the outcome of competition among Cladocera as a function of food supply (Romanovsky and Feniova, 1985).

However, Gliwicz (1990) tried to predict the outcome of competition among zooplankton by measuring Daphnia threshold food requirements in young individuals only (2-6 days old). As his approach aimed to predict events at a population level, his measurements implicitly assumed similar sensitivities to starvation of juveniles and adults. Earlier, Threlkeld (1976) proposed a model of survival time of zooplankton as a function of weight-specific respiration rate and the fraction of prestarvation body weight under conditions of starvation. Testing of that model suggested a possibility that aged adults may be very sensitive to starvation as well.

Assuming a strong correlation between starvation and increased population density via reduced food concentration, we can summarize the different views by 
proposing three types of dependencies to predict the responses of mortality to an increase in population density (or to a decline in per capita food supply): (i) the mortality of adults remains constant or increases at a slower rate than that of juveniles (Romanovsky and Feniova, 1985), (ii) the mortalities of both juveniles and adults increase at the same rate (a view implicit in Gliwicz, 1990), (iii) the mortality of adults increases while the mortality of juveniles remains constant or increases at a slower rate. These three alternatives would probably cover major conceivable cases.

Below, we re-analyse an experiment on competition between Ceriodaphnia pulchella Sars and Diaphanosoma brachyurum (Lievin) described earlier by Matveev (1987a). In this experiment Diaphanosoma was invariably outcompeted under conditions of epilimnion water of meso-eutrophic Lake Glubokoe, Moscow Region. Our objective here was to elucidate the demographic mechanism of that competitive exclusion. We analysed densitydependencies of three finite population rates: fecundity rate, as well as juvenile and adult death rates. Firstly, we tried to find out if these rates were correlated to population densities. As birth rates and death rates are normally delayed functions of density (Matveev, 1985, 1987b) we used time lag analysis. Secondly, by comparing the slopes of regressions of the three rates we determined which of them were more sensitive to changes in density and identified which age group is more vulnerable in competition.

\section{Method}

The experiment was conducted in 0.6 I stoppered flasks with epilimnial lake water void of zooplankton ( $95-\mu \mathrm{m}$ filtration). The filtration provided a natural spectrum of phytoplankton as food for cladocerans in the range of cell sizes of 2$40 \mu \mathrm{m}$; there were no larger cells. The algae were represented by two categories of naked flagellates and three categories of Chlorococcales, the densities of which were monitored throughout the experiment (Matveev, 1987a). The estimated overlap in feeding niches of Ceriodaphnia and Diaphanosoma was the highest possible and symmetrical according to MacArthur-Levins' index of overlap (Matveev, 1987a). There were five control flasks for Ceriodaphnia and five for Diaphanosoma, which were inoculated with 1-2-instar juveniles. These flasks had isolated populations with starting densities of $2,4,6,8$ and 10 animals flask $^{-1}$ for each species. In five experimental flasks, where mixed populations were created, the starting densities of the two competitors varied yielding the ratios of $2: 10,4: 8,6: 6,8: 4$ and 10:2 animals flask ${ }^{-1}$ (one replicate per treatment). Thus, for a given species, the starting density was the same in the control flask and the corresponding experimental flask. Such a design implies that under the null hypothesis of no competition, there will be no difference between the mean size of an isolated population for the growing period and of a population in the presence of an alien species. The effect of competition is inferred when the difference is significant or/and one of the populations becomes extinct. The experiment was started on 9 June 1983, and was run for 77 days at a temperature of $20 \pm 2^{\circ} \mathrm{C}$. Every $3-4$ days animals and eggs were counted, the 
water changed and the corpses removed, counted and measured to distinguish between juveniles and adults. By the end of a 4-day interval between censuses, when animal densities were at their maximum, oxygen in the flasks was not depleted, remaining above $9.4 \mathrm{mg} \mathrm{l}^{-1}$. For more details of the experiment see Matveev (1987a).

Finite rates of mortality and fecundity were estimated using the formulae:

$$
d_{\mathrm{j}}=D_{\mathrm{j}} /\left(N_{\mathrm{j}} \Delta t\right), d_{\mathrm{a}}=D_{\mathrm{a}} /\left(N_{\mathrm{a}} \Delta t\right), f=F /\left(N_{\mathrm{a}} \cdot T\right)
$$

where $d_{\mathrm{j}}=$ finite death rate of juveniles, $d_{\mathrm{a}}=$ finite death rate of adults, $f=$ finite rate of fecundity, $D_{j}=$ number of juvenile corpses found by the end of the interval between consecutive censuses ( $\Delta t=3$ or 4 days), $N_{\mathrm{j}}=$ number of live juveniles (at the beginning of $\Delta t), D_{\mathrm{a}}=$ number of adult corpses by the end of $\Delta t, N_{\mathrm{a}}=$ number of live adults, $F=$ total number of eggs, $T=$ egg development time, determined from the dependence on the temperature (Bottrell et al., 1976).

To determine density-dependencies in both mixed and isolated cultures, we regressed $d_{\mathrm{j}}, d_{\mathrm{a}}$ and $f$ versus an estimate of population density introducing time lags $(\tau)$. For mixed cultures, total biomass of two competitors was used as an estimate of population density. The biomasses were calculated according to assumed constant mean weights of juveniles and adults of each of the two cladocerans. The weights were assessed on the basis of body length measurements and using length-weight relationships (Bottrell et al., 1976). In singlespecies cultures, the calculation of biomasses by multiplying population numbers by constant weights did not make density estimates more accurate. Therefore, density estimates for isolated populations were total numbers only.

Prior to calculating cross-correlations, the numbers of eggs, live and dead adults and juveniles were smoothed using a three point moving average to reduce noise in the data sets. Because time intervals between measurements were not constant, we weighted the points inversely according to the corresponding time intervals. The values of density estimates between days of censuses, necessary for cross-correlations, were obtained by cubic spline interpolation. We used Spearman rank correlation coefficients, but also always computed parametric product-moment correlation coefficients. Both gave very similar results. This might mean that the assumptions underlying parametric tests were not severely violated. After a preliminary scanning for time lags that maximize the absolute value of correlation using 0.5-day time step, we determined population delays with a numerical accuracy of 0.005 days so that the error in time delay resulting from the computations is negligible compared to the error resulting from experimental noise. The range of time delays considered was restricted to biologically meaningful intervals ( $<2$ weeks, Matveev, 1983). By this means we avoided pseudocorrelations which could arise (e.g. between phases of population increase and decrease).

Because all finite rates contained an estimate of density in the denominator and these rates were compared with population densities, there was a possibility of obtaining misleading autocorrelations rather than true biological density- 
dependencies. To overcome this problem, we compared a cross-correlation curve for a given rate with a cross-correlation curve where the numerator of the rate formula was substituted by 1 ('placebo'). For example, if $D_{\mathrm{j}} / N_{\mathrm{j}} \Delta t$ ) was compared with $N(t-\tau)$ for a density-dependence at a time lag $\tau$, a comparison was also made between $1 /\left(N_{\mathrm{j}} \Delta t\right)$ and $N(t-\tau)$. In the computed placebo crosscorrelation a constant value of 1 had no effect on the variation of the rate itself. In the normal cross-correlation the numerator was a variable which affected the variation of a given rate. By comparing placebo and normal cross-correlations one could infer the influence of the normal numerator on the rate and its variation. We accepted a normal cross-correlation as valid, reflecting biological density dependence, if it differed significantly from placebo cross-correlation (e.g. Figure 1). If the two resulting curves had not differed, the detection of a density-dependent relationship for that rate was considered to be impossible.

A time lag corresponding to a maximum (by the absolute value) of a crosscorrelation curve was taken to construct a density-dependent regression. The estimates of the slope of the regression remained virtually the same if 1-2 day shifts in the time lag were allowed.

Even when replicated, each experimental population of Cladocera has a unique history of development (Slobodkin, 1954). This can make impossible the analysis of density-dependence if it is based on the pooled data from several populations. Therefore, like in previous studies (Matveev, 1985, 1987b), we analysed each species population separately. For 77 days of the experiment, the maximal number of data points per experimental unit could reach 22. However,

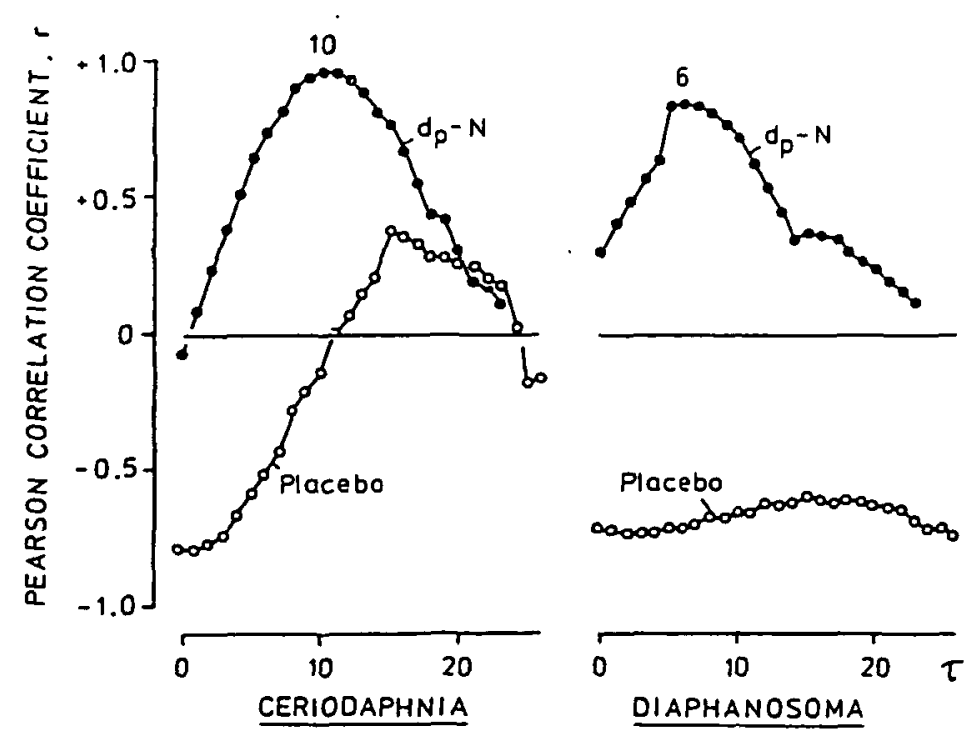

Fig. 1. Cross-correlation curves for two isolated populations of Ceriodaphnia and Diaphanosoma. Black circles: cross-correlations for the observed dependencies of overall finite death rates as functions of density $\left(d_{p}-N\right)$ at different time lag shifts ( $\tau$-values in days on abscissa). Hollow circles: the corresponding 'placebo'-curves (see explanations in the text). Figures above curves are the values in days of time lags found. 
because Diaphanosoma in mixed cultures approached extinction level long before the experiment was terminated, this number was normally reduced. Linear regressions were estimated for all finite rates as functions of density. In the case of fecundity, a better fit might have been obtained by a non-linear approximation; however, for the sake of comparison with the slopes of other rates, fecundity relationships were also linearly fitted. The significance of the difference between regression slopes was estimated using a $t$-test (Zar, 1984).

\section{Results}

In five mixed cultures Diaphanosoma was outcompeted by Ceriodaphnia irrespective of initial relative frequencies (Matveev, 1987a). Two treatments of mixed cultures representing the extreme cases of competitors' initial ratio are given in Figure 2 (left). In isolated cultures, both Ceriodaphnia and Diaphanosoma persisted until the experiment was terminated (Figure 2, right). Therefore, interspecific competition was apparently strong.

The introduction of a time lag provided to be crucial for identifying densitydependent relationships. This is illustrated by the example of the overall finite death rate, $d_{\mathrm{p}}$ of a population as a function of its density $\left(d_{\mathrm{p}}-N\right)$ in isolated cultures (Figure 1). In Ceriodaphnia, the assumption of no time lag in the responses of mortality yields a value of correlation coefficient close to zero
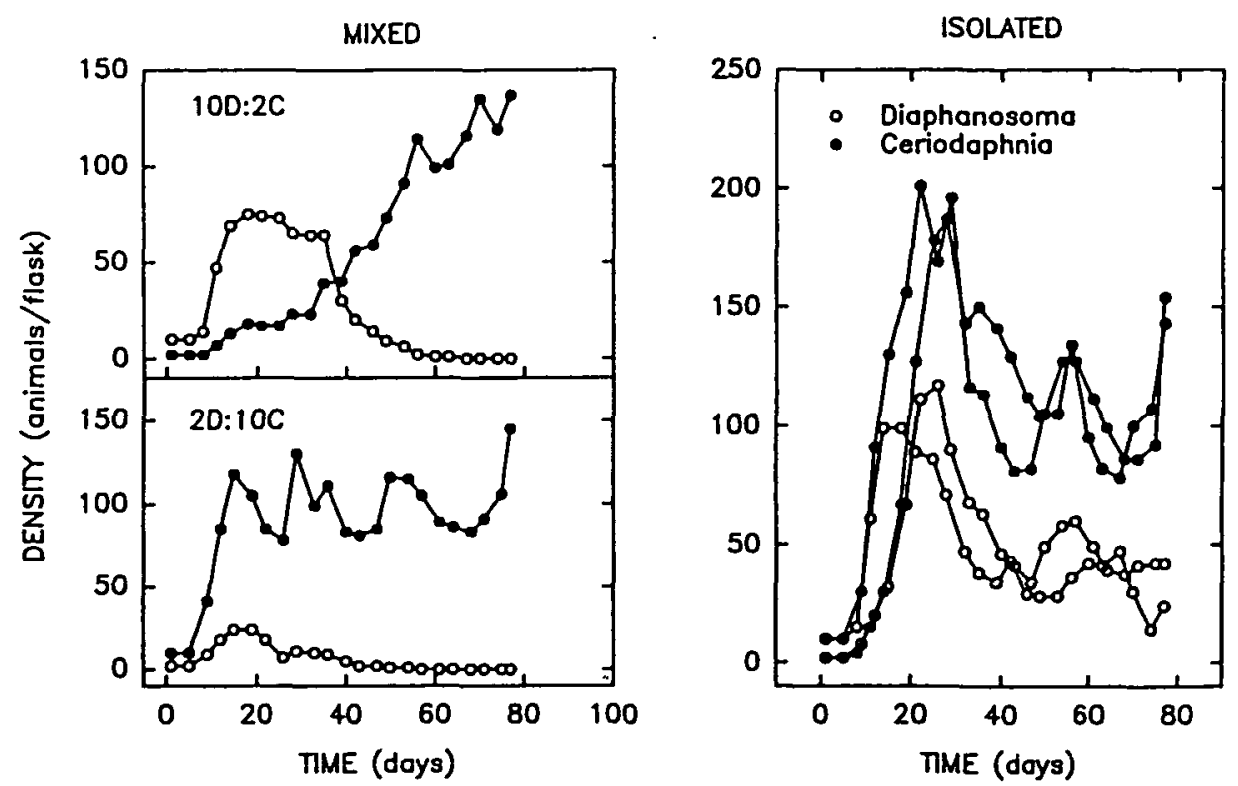

Fig. 2. An example of the population growth of Diaphanosoma and Ceriodaphnia in mixed versus isolated cultures of the competition experiment with varying starting frequencies. In mixed cultures, the starting numbers of competitors are 10 animals of Diaphanosoma + two animals of Ceriodaphnia (10D:2C, upper plot), or two animals of Diaphanosoma + 10 animals of Ceriodaphnia (2D:10C, lower plot). In isolated cultures, the starting densities are 2D, 10D, 2C, $10 \mathrm{C}$. 
(Figure 1, left, black circles). An increase in the magnitude of time lag leads to an increase in the correlation coefficient. At 10-day time lag a peak is reached, where $92 \%$ of variance in death rate is explained by the density (Figure 1, left, black circles for Ceriodaphnia). A similar 'humped' cross-correlation was obtained for Diaphanosoma (Figure 1, right, black circles). Here the time lag maximizing the correlation between death rate and density was equal to 6 days ( $72 \%$ of explained variance). The placebo curves (Figure 1, hollow circles) do not coincide with those based on original data indicating that the real dependencies for death rates are well separated from autocorrelations.

In isolated cultures of Ceriodaphnia, adult and juvenile death rates taken separately, as well as fecundity rate, were also delayed functions of density. As maximal absolute values of cross-correlation curves suggest (Figure 3, left), there was approximately a 3-day time lag in responses of juvenile death rate, and a 10-day time lag in responses of adult death rate to density. Fecundity rate responded to density changes with a 12-day lag.

The comparison of the three finite rates regressed on population density at the time lags found (Figure 3, right) revealed that fecundity rate was a decreasing function of density, while both death rates were increasing functions. Secondly, as density increased, a drop in fecundity rate was much sharper than an increase in both death rates. Thirdly, the death rate of juveniles grew faster with density

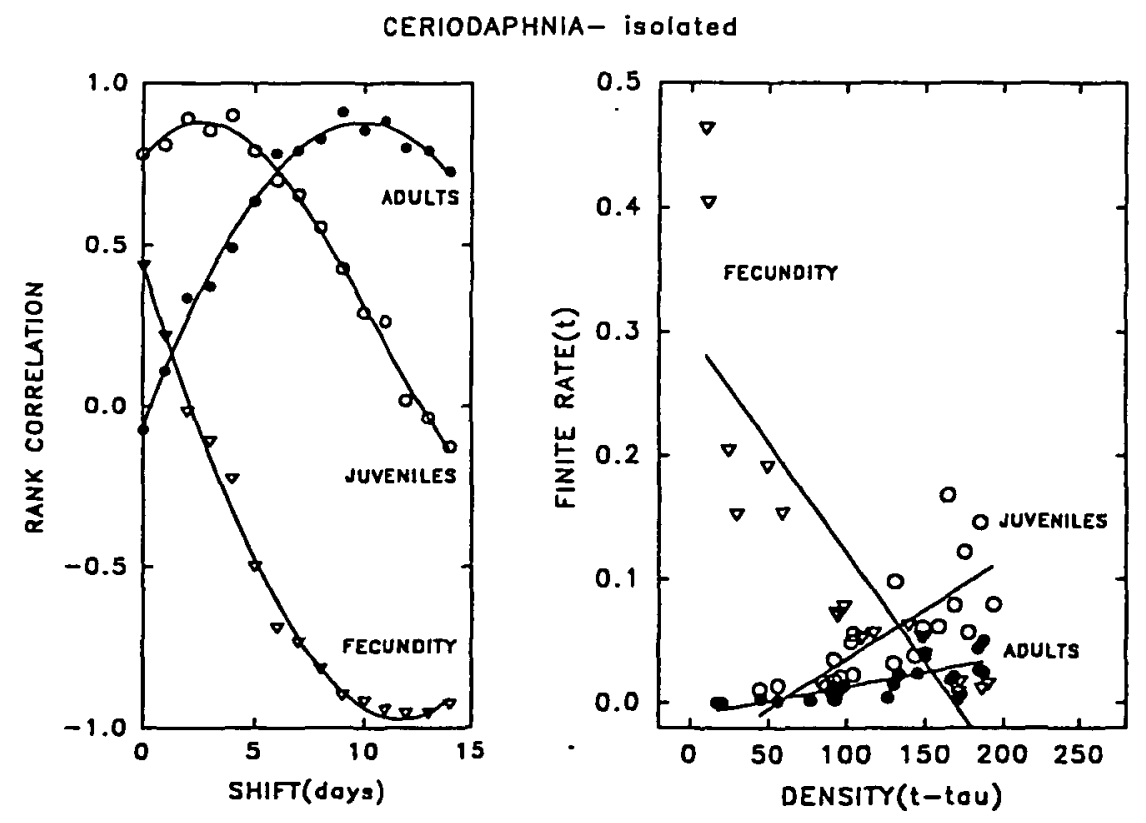

Fig. 3. Density regulation in an isolated culture of Ceriodaphnia. Left: Cross-correlation curves for juvenile death rate ('juveniles'), adult death rate ('adults') and fecundity rate ('fecundity') correlated to population density at different time lag shifts. Right: The resulting regressions of the three rates on density (animals flask ${ }^{-1}$ ) at time lags found ( 3 days in juveniles, 10 days in adults and 12 days in fecundity). The regression coefficients: fecundity, $b=-1.77 \times 10^{-3}\left(r^{2}=0.73, P<0.001\right)$; juveniles, $b=8.03 \times 10^{-4}\left(r^{2}=0.58, P<0.001\right)$; adults, $b=2.32 \times 10^{-4}\left(r^{2}=0.68, P<0.001\right)$. 
than the death rate of adults $(P<0.001, t$-test for slopes). Thus, fecundity was more sensitive to density regulation than juveniles, while juveniles were more sensitive than adults (Figure 3 , right).

Similar time lags and sensitivity to regulation were found in juveniles and fecundity of isolated cultures of Diaphanosoma. Juvenile responses to density were delayed by $\sim 3$ days, while fecundity rate was delayed by 12 days (Figure 4 , left). In adults, values of the positive area of the cross-correlation curve were not significant (Figure 4, left). This did not allow inference of the mode of densitydependence, so the corresponding regression was not constructed. As in Ceriodaphnia fecundity rate was more sensitive to density changes than juvenile death rate (Figure 4, right).

In mixed cultures, cross-correlations for Ceriodaphnia were similar to those in isolated cultures. Diaphanosoma in mixed cultures showed a strong densitydependence of adult death rate at zero time lag, a weak juvenile densitydependence at 9-day time lag and a 10-day delay in the responses of fecundity (Figure 5). In mixed cultures, the slopes of regressions of death rates on density differed between the two competitors in a contrasting way: while in Ceriodaphnia the slope for juveniles was steeper than the slope for adults $(P<0.05)$,

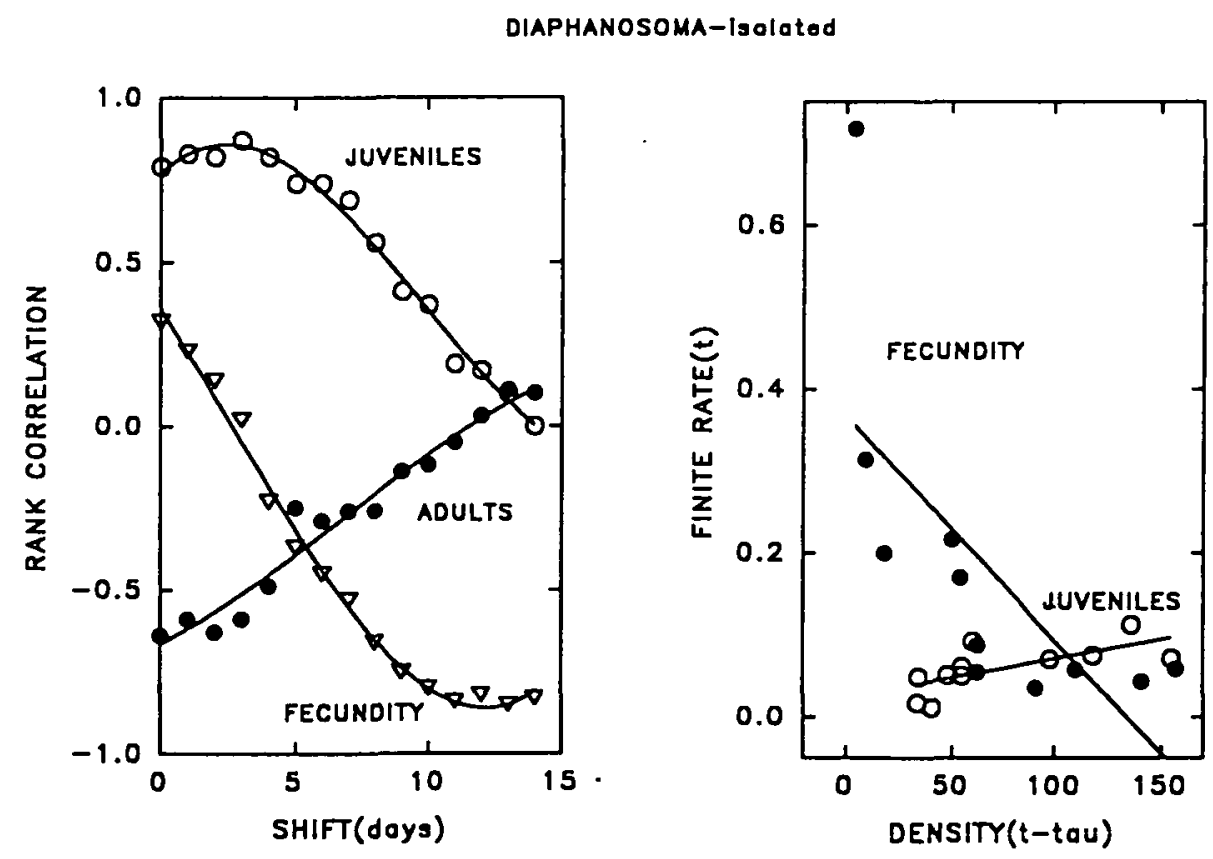

Fig. 4. Density regulation in an isolated culture of Diaphanosoma. Left: Cross-correlation curves for juvenile death rate ('juveniles'), adult death rate ('adults') and fecundity rate ('fecundity') correlated to population density at different time lag shifts. Right: The resulting regressions of the two rates on density (animals flask ${ }^{-1}$ ) at time lags found ( 3 days in juveniles, 12 days in fecundity). The regression coefficients: fecundity, $b=-2.74 \times 10^{-3}\left(r^{2}=0.49, P<0.02\right)$; juveniles, $b=4.70 \times 10^{-4}\left(r^{2}=\right.$ $0.47, P<0.02$ ). 


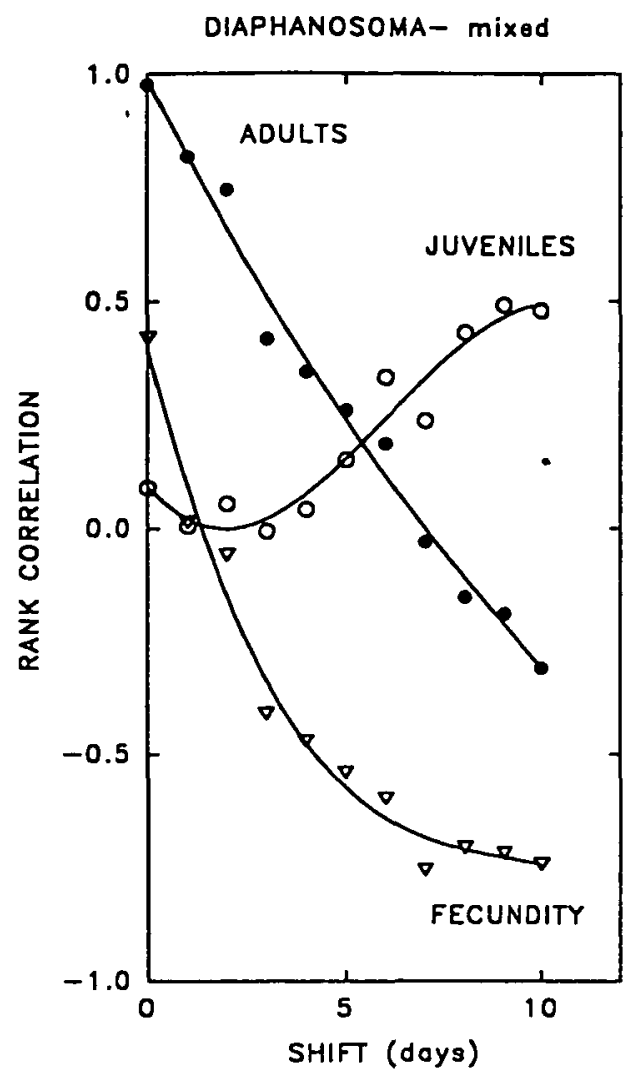

Fig. 5. Cross-correlation curves for Diaphanosoma population in a mixed culture. Adult death rate ('adults'), juvenile death rate ('juveniles') and fecundity rate ('fecundity') are correlated to density at different time lag shifts.

in Diaphanosoma adult death rate grew faster than juvenile death rate as density increased $(P<0.01)$ (Figure 6).

To understand the demographic peculiarities of Diaphanosoma exclusion, we compared the slopes of the three finite rates as functions of density between two competitors in mixed cultures. The slopes for fecundity did not differ significantly between Ceriodaphnia and Diaphanosoma (Figure 7A). As density increased, juvenile death rate of Diaphanosoma did not grow faster than that of Ceriodaphnia (Figure 7B). However, adult death rate of Diaphanosoma increased with density much faster than adult death rate of Ceriodaphnia: the difference in slopes was highly significant $(P<0.005)$ (Figure $7 C$ ). Thus, Diaphanosoma was apparently outcompeted through elevated mortality of adults.

\section{Discussion}

A test for food exploitation showed that Diaphanosoma and Ceriodaphnia could diminish the algal concentrations in the analysed cultures by at least an order of 


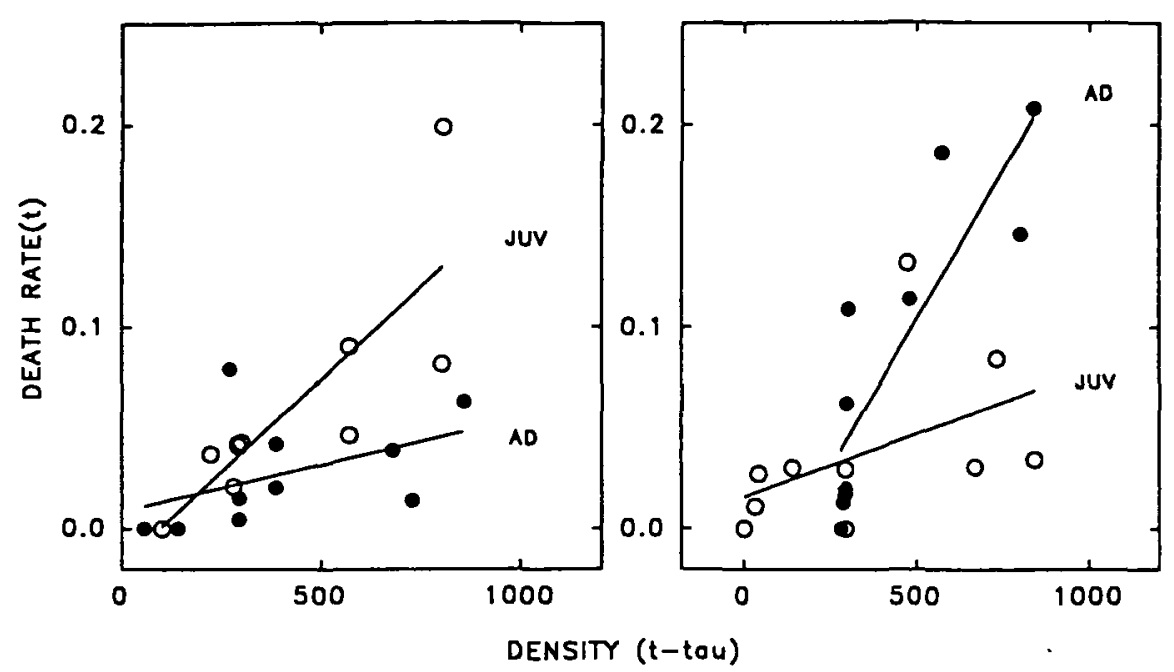

Fig. 6. The dependencies of finite death rates on total density ( $\mu \mathrm{g}$ dry weight flask ${ }^{-1}$ ) in two competitors in a mixed culture. Juveniles ('juv') versus adults ('ad'). The regression coefficients in Ceriodaphnia: juveniles, $b=1.84 \times 10^{-4}\left(r^{2}=0.67, P<0.01\right)$, adults, $b=4.63 \times 10^{-5}\left(r^{2}=\right.$ $0.20, P>0.10)$; in Diaphanosoma: juveniles, $b=5.5 \times 10^{-5}\left(r^{2}=0.17, P>0.10\right)$, adults, $b=$ $2.92 \times 10^{-4}\left(r^{2}=0.73, P<0.005\right)$.

magnitude in 3 days (Matveev, 1987a). Present analysis suggests that under such food limitation fecundity seems to be the most sensitive density-dependent parameter. This agrees with a similar finding for the populations of daphnids and Diaphanosoma competing for food in a North American lake (Kerfoot et al., 1985). However, fecundity did not seem to be the way through which Diaphanosoma was driven to extinction in our experiment: there was no difference between the slopes of fecundity regressions in Diaphanosoma versus Ceriodaphnia. Of the three aforesaid conceivable patterns of mortality regulation, the case of equal sensitivity of juveniles and adults to density did not seem to hold in either of the two competitors. There have always been differences between juvenile and adult mortality in our experiments. In Ceriodaphnia, juvenile mortality was regulated more strongly than adult mortality in both isolated and mixed cultures. Thus, the case of Ceriodaphnia may support the view that juveniles of some cladocerans are more vulnerable than adults to density effects such as food limitation. But neither Ceriodaphnia nor Diaphanosoma seemed to show that adult death rate remained constant along the gradient of animal densities/food concentrations in contrast to the assumption of Romanovsky and Feniova (1985). In Diaphanosoma, the species which have been outcompeted in all our cultures, adult mortality increased much more rapidly with density than juvenile mortality. Pairwise comparisons of the two competitors in mixed cultures gave a clear indication that the elevated mortality of Diaphanosoma adults was responsible for its extinction, while juvenile death rate in both species was similar. Alternatively, the extinction of 

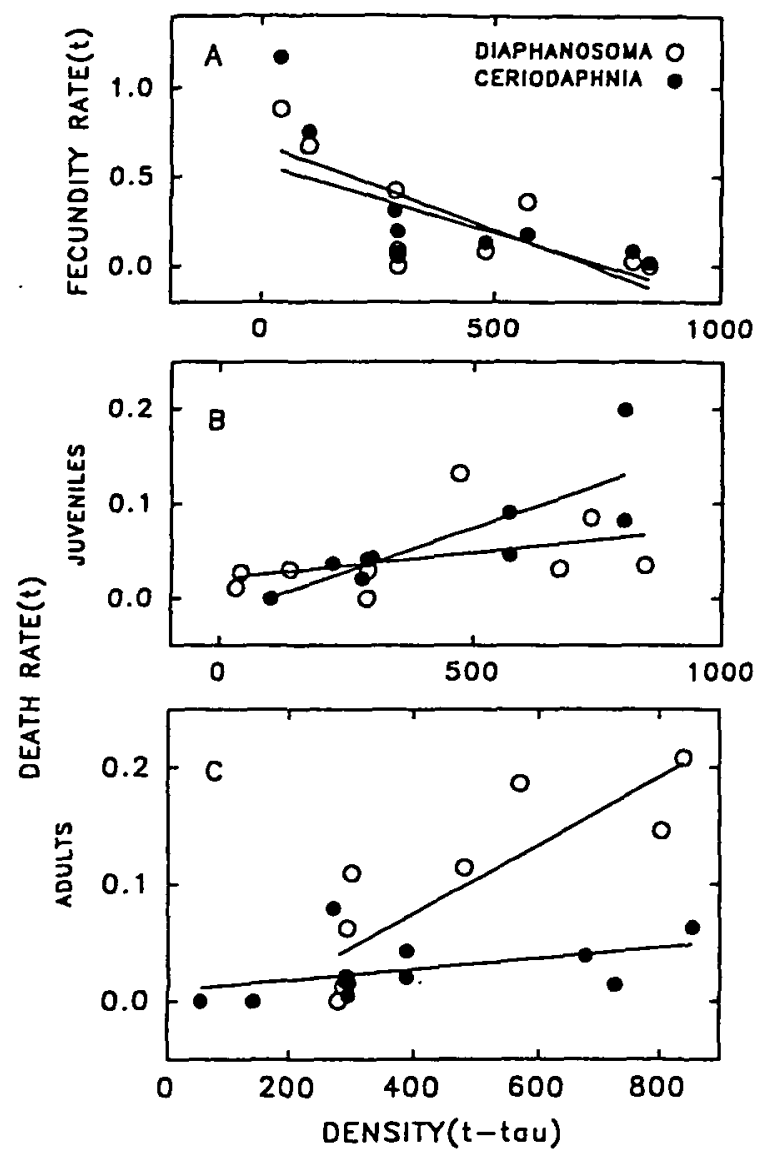

Fig. 7. The dependencies of three population finite rates on density ( $\mu \mathrm{g}$ dry weight flask ${ }^{-1}$ ) in two competitors in a mixed culture. A. Finite fecundity rates (Diaphanosoma: $b=-7.62 \times 10^{-4}, r^{2}=$ $0.44, P<0.05$; Ceriodaphnia: $\left.b=-9.55 \times 10^{-4}, r^{2}=0.48, P<0.05\right)$. B. Finite death rates of juveniles (Diaphanosoma: $b=5.5 \times 10^{-5}, r^{2}=0.17, P>0.10$; Ceriodaphnia: $b=1.84 \times 10^{-4}$, $r^{2}=0.67, P<0.01$ ). C. Finite death rates of adults (Diaphanosoma: $2.92 \times 10^{-4}, r^{2}=0.73$, $P<0.005$; Ceriodaphnia: $b=4.63 \times 10^{-5}, r^{2}=0.20, P>0.10$ ).

Diaphanosoma in the present experiment cannot be explained by its higher vulnerability to laboratory handling, firstly, because handling-induced mortality is density-independent, while we found the dependence of Diaphanosoma death rate on density, and secondly because in a similar experiment on competition with Daphnia, Diaphanosoma could have been a superior competitor (Matveev, 1987b). Thus, the present study suggests that the exclusion of an inferior competitor through elevated mortality of juvenilies (Neill, 1975; Lynch, 1978) was not the case in competition between Ceriodaphnia and Diaphanosoma.

At first sight, the suggestion of extinction through elevated mortality of adults seems paradoxical. However, it finds support in the view that Cladocera may have increased mortality in aged individuals (Threlkeld, 1976). Moreover, Diaphanosoma is known to be a genus which seems to flourish best at high 
temperatures; it has a number of tropicopolitan species (Hutchinson, 1967). In the temperate lakes of the northern hemisphere, it normally yields one summer population peak and then disappears until the next year (Hutchinson, 1967). Its life cycle therefore may be adapted for quick population performance during the warm season. This does not imply a strategy for adults to have high survival during extended periods of time. On the contrary, with a relatively short life cycle under conditions of food limitation adults can make a higher investment to reproduction at the expense of their subsequently reduced survival. That the population dynamics of Diaphanosoma in lakes to a considerable extent is determined by food limitation has been suggested many times (Hutchinson, 1967; Matveev, 1983, 1991; Romanovsky and Feniova, 1985; DeMott, 1989). Therefore, the models of competition universally assuming higher juvenile than adult sensitivity to crowding and starvation are probably applicable only to a limited number of cases.

Our analysis suggests that high vulnerability of juveniles to starvation and crowding is not a universal demographic mechanism by which a cladoceran population may be outcompeted. Adults may be more vulnerable to these conditions. It is probably worth conducting the experiments specifically designed to compare mortality of adults and juveniles in Diaphanosoma and other similar species. The answer to the question why demographic mechanisms of competitive exclusion vary from species to species may probably be found in comparisons of life cycles of Cladocera.

\section{Acknowledgements}

This research was funded by the USSR Academy of Sciences and by a stipend of the Max Planck Society to V.M. We are grateful to Winfried Lampert for support and encouragement, to Lilian Matveeva, Bill DeMott, John Pontin and two referees for critical comments on the manuscript.

\section{References}

Bottrell,H.H., Duncan,A., Gliwicz,Z.M., Grygierek,E., Herzig,A., Hillbricht-Ilkowska,A., Kurasawa,H., Larsson,P. and Weglenska,T. (1976) A review of some problems in zooplankton production studies. Norw. J. Zool., 24, 419-456.

DeMott,W.R. (1989) The role of competition for resources in phytoplankton succession. In Sommer,U. (ed.), Plankton Ecology. Succession in Plankton Communities. Springer Verlag, Berlin, pp. 195-252.

Gliwicz,Z.M. (1990) Food thresholds and body size in cladocerans. Nature, 343, 638-640.

Hutchinson,G.E. (1967) A Treatise on Limnology. Volume 2. Introduction to Lake Biology and the Limnoplankton. Wiley, New York.

Kerfoot,W.C., DeMott,W.R. and Levitan,C. (1985) Nonlinearities in competitive interactions: Component variables or system response? Ecology, 66, 959-965.

Lynch,M. (1978) Complex interactions between natural coexploiters-Daphnia and Ceriodaphnia. Ecology, 59, 552-564.

Matveev,V.F. (1983) Estimating competition in cladocerans using data on dynamics of clutch size and population density. Int. Rev. Ges. Hydrobiol., 68, 785-798.

Matveev,V. (1985) Competition and population time lags in Bosmina (Cladocera, Crustacea). Int. Rev. Ges. Hydrobiol., 70, 491-508.

Matveev,V.F. (1987a) Competitive exclusion in planktonic cladocerans of different families under conditions of a resource spectrum. Izv. Akad. Nauk SSSR, Ser. Biol., N5, 728-736. 


\section{V.Matveev and W.Gabriel}

Matveev,V.F. (1987b) Effect of competition on the demography of planktonic cladoceransDaphnia and Diaphanosoma. Oecologia (Berlin), 74, 468-477.

Matveev,V. (1991) Exploitative and interference competition among planktonic crustaceans in a subtropical lake. Arch. Hydrobiol., 123, 53-68.

Neill,W.E. (1975) Experimental studies of microcrustacean competition, community composition and efficiency of resource utilization. Ecology, 56, 809-826.

Romanovsky,Y.E. and Feniova,I.Y. (1985) Competition among Cladocera: effect of different levels of food supply. Oikos, 44, 243-252.

Slobodkin, L.B. (1954) Population dynamics in Daphnia obtusa Kurz. Ecol. Monogr., 24, 69-88.

Tessier,A.J., Henry, L.L., Goulden,C.E. and Durand,M.W. (1983) Starvation in Daphnia: Energy reserves and reproductive allocation. Limnol. Oceanogr., 28, 667-676.

Threlkeld,S.T. (1976) Starvation and the size structure of zooplankton communities. Freshwater Biol., 6, 489-496.

Zar,J.H. (1984) Biostatistical Analysis. Prentice-Hall, Englewood Cliffs, NJ.

Received on December 16, 1993; accepted on March 31, 1994 